\title{
Postdischarge rheumatic and musculoskeletal symptoms following hospitalization for COVID-19: prospective follow-up by phone interviews
}

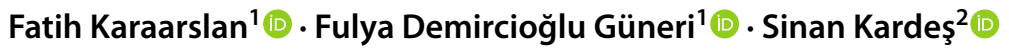 \\ Received: 24 March 2021 / Accepted: 29 April 2021 / Published online: 12 May 2021 \\ (c) The Author(s), under exclusive licence to Springer-Verlag GmbH Germany, part of Springer Nature 2021
}

\begin{abstract}
To describe the rheumatic and musculoskeletal symptoms at hospitalization as well as their persistence/severity after discharge with coronavirus disease 2019 (COVID-19) and to identify whether age, sex, body mass index (BMI), and length of hospital stay are associated with persistence of these symptoms. In this single-center cohort study, comprising 300 participants, two phone interviews were conducted (2-week and 1-month after hospitalization) and symptoms were queried with a standardized form. This form included musculoskeletal symptoms and other COVID-19 symptoms. Considering all symptoms (musculoskeletal and other), 100.0\%, 86.7\%, and $72.0 \%$ of patients reported one or more symptoms, at hospitalization, 2-week, and 1-month, respectively. Considering only musculoskeletal symptoms, 92.3\%, 72.7\%, and 56.3\% of patients reported any musculoskeletal symptom at hospitalization, 2-week, and 1-month, respectively. The musculoskeletal symptoms were fatigue (44.3\% of patients reported), back pain (22.7\%), arthralgia (22.0\%), myalgia (21.0\%), low back pain (16.3\%), and neck pain (10.3\%); the other symptoms were shortness of breath (26.3\%), loss of taste (15.0\%), cough (14.0\%), loss of smell (12.3\%), loss of appetite (10.3\%), headache (8.7\%), sore throat (3.0\%), diarrhea (1.3\%), dizziness (1.3\%), and fever $(0.3 \%)$ at 1 -month. Increasing BMI was associated with higher odds of persistence of fatigue (OR: 1.08, 1.03 to 1.13), myalgia (OR: $1.08,1.01$ to 1.14 ), and arthralgia (OR: 1.07, 1.02 to $1.14, p=0.012$ ) at 1 -month. Nearly three-quarters reported one or more symptoms, with more than half of patients reported any musculoskeletal symptom at 1 month. The most common musculoskeletal symptom was fatigue, followed by back pain, arthralgia, myalgia, low back pain, and neck pain. The persistence of fatigue, myalgia, and arthralgia was related to BMI. The study results increase our understanding of the spectrum of COVID-19, which, in turn, may lead to more efficient and better care for COVID-19 survivors.
\end{abstract}

Keywords COVID-19 Post-Acute Sequelae of SARS-CoV-2 infection (PASC) $\cdot$ Fatigue $\cdot$ Myalgia $\cdot$ Arthralgia $\cdot$ Pain $\cdot$ Rheumatic symptoms $\cdot$ Musculoskeletal symptoms

Sinan Kardeş

sinan.kardes@istanbul.edu.tr

Fatih Karaarslan

fatih.karaarslan@sbu.edu.tr

Fulya Demircioğlu Güneri

fuliad@hotmail.com

1 Department of Medical Ecology and Hydroclimatology, Gulhane Training and Research Hospital, University of Health Sciences, Ankara, Turkey

2 Department of Medical Ecology and Hydroclimatology, Istanbul Faculty of Medicine, Istanbul University, Istanbul, Turkey

\section{Introduction}

The novel coronavirus, severe acute respiratory syndrome coronavirus 2 (SARS-CoV-2), which causes a disease named coronavirus disease 2019 (COVID-19), emerged in China in December 2019 [1, 2]. Its rapid worldwide spread leading to an unexpected pandemic has challenged as a global health threat and considerably impacted nearly all aspects of the life of individuals as well as healthcare systems [3-8]. Globally, over 114 million confirmed cases with more than 2.5 million deaths from COVID-19 have been documented as of March 2021 [9].

The initial symptoms of COVID-19 have been well documented. In a report among 373,883 COVID-19 cases with known symptom status, cough, fever, myalgia, headache, 
shortness of breath, sore throat, diarrhea, nausea/vomiting, loss of smell or taste, abdominal pain, and runny nose were described in the descending order of frequency [10]. Furthermore, a meta-analysis addressing the rheumatic manifestations of COVID-19 found a pooled estimation of $19 \%$ for myalgia and $32 \%$ for fatigue as an initial symptom of COVID-19 [11]. However, these studies investigated rheumatic and musculoskeletal symptoms only as of the presence/absence of fatigue, myalgia, and arthralgia.

To the knowledge of the authors, information on detailed documentation of rheumatic and musculoskeletal symptoms at hospitalization as well as their severity/persistence after discharge with COVID-19 is scarce [12-17]. More granular data for rheumatic and musculoskeletal symptoms would increase our understanding of the spectrum of COVID-19, which, in turn, leads to more efficient and better care for COVID-19 survivors. The aim of the present study, therefore, was to describe postdischarge rheumatic and musculoskeletal symptoms following hospitalization for COVID-19 and to identify whether age, sex, body mass index (BMI), and length of hospital stay are associated with the persistence of these symptoms.

\section{Methods}

\section{Study design}

The study was a single-center cohort study with prospective follow-up by phone interviews. It was conducted in accordance with the Declaration of Helsinki. Ethical approval for the study was granted by the institutional ethics committee. Informed verbal consent was obtained from all participants by phone before proceeding to the survey.

\section{Study setting}

The study was performed at Gulhane Training and Research Hospital, a Tertiary Hospital.

\section{Participants}

Participants who were between 18 and 70 years of age, and discharged following hospitalization for COVID-19 between November 18, 2020, and January 30, 2021, were eligible for inclusion. Participants who received intensive care unit (ICU) care at any time during the hospitalization were excluded.

\section{Data collection}

A total of two phone interviews were conducted (2-week and 1-month after hospitalization). Demographic and baseline characteristics (including age, sex, height, weight, educational level, employment status, comorbidities), initial symptoms at hospitalization (musculoskeletal and others), and length of hospital stay were queried and obtained at the 2 -week phone survey. Symptoms of 2-week and 1-month were queried and obtained at 2-week and 1-month phone survey, respectively, with a standardized form. This form included musculoskeletal symptoms (fatigue, myalgia, low back pain, back pain, and neck pain) and other COVID19 symptoms (fever, cough, loss of appetite, shortness of breath, diarrhea, sore throat, headache, dizziness, loss of taste, and loss of smell). The severity of each musculoskeletal and other COVID-19 symptom was queried with a fivelevel Likert scale (i.e. none; mild; moderate; severe; and very severe).

\section{Statistical methods}

The characteristics were analyzed with descriptive statistics, and presented as mean and standard deviation for continuous variables and as frequency and percentage for categorical variables. Generalized estimating equations with a binary logistic model was used to assess the association of age, sex, BMI, and length of hospital stay with the presence of fatigue, myalgia, and arthralgia at 1 month. The sample size was calculated according to the formula provided elsewhere [18]: the number of independent variables was 4 , it was estimated a total of 300 participants are needed. All statistical analyses were carried out using Statistical Package for the Social Sciences (SPSS) version 21.0 (IBM Corp.). A twotailed $p$ value $<0.05$ was considered statistically significant. The presentation of statistical results was informed by the recent review [19].

\section{Results}

The demographic and clinical characteristics of the study population comprising 300 participants are presented in Table 1 . The majority were male $(60 \%)$, the mean age was 53 years, $65 \%$ had at least one comorbidity, and the mean length of hospital stay was 8 days.

At hospitalization, all patients reported one or more symptoms, with $92.3 \%$ reported any musculoskeletal symptom and $98.7 \%$ reported any other COVID-19 symptom. The musculoskeletal symptoms were fatigue $(87.3 \%$ of patients reported), myalgia (63.3\%), arthralgia (59.3\%), back pain (54.0\%), low back pain (44.7\%), and neck pain (26.7\%); the other COVID-19 symptoms were shortness of breath (76.7\%), loss of appetite $(71.7 \%)$, cough $(67.7 \%)$, fever $(65.3 \%)$, loss of taste $(53.0 \%)$, loss of smell $(43.3 \%)$, headache $(39.3 \%)$, sore throat $(30.3 \%)$, diarrhea $(21.3 \%)$, and dizziness $(3.7 \%$ ), in the descending order of frequency (Fig. 1, 
Fig. 1 Percentage of hospitalization and postdischarge symptoms. Percentages are among all patients $(n=300)$. The symptoms were listed in the descending order of percentages at 1-month in musculoskeletal symptoms and other COVID-19 symptoms categories
-Hospitalization $\quad$ 2-week 1-month

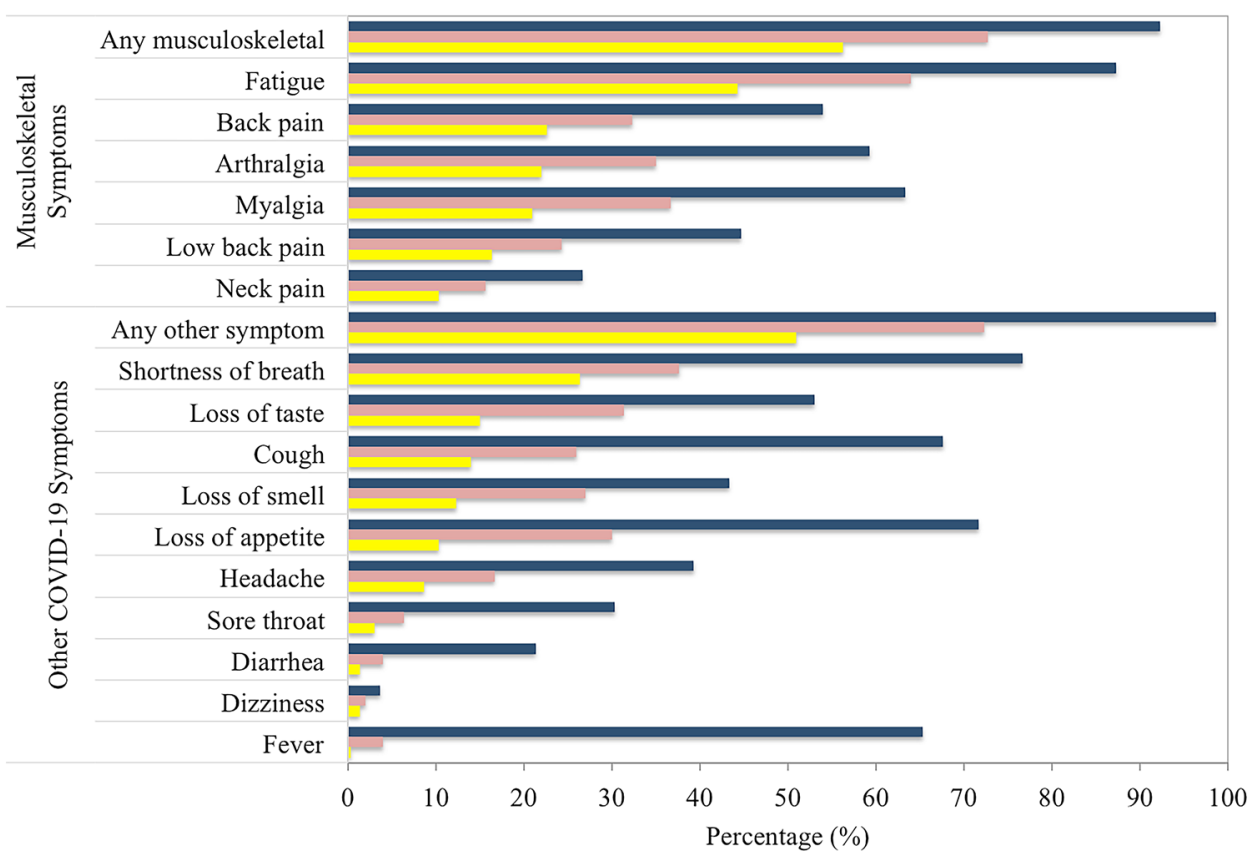

Table 2). Myalgia and arthralgia were reported mostly as widespread than local; if these were local the most frequent regions were lower leg and knee, respectively (Table 3).

At 2-week, $86.7 \%$ reported one or more symptoms, with $72.7 \%$ reported any musculoskeletal symptom and $72.3 \%$ reported any other COVID-19 symptom. The musculoskeletal symptoms were fatigue ( $64.0 \%$ of patients reported), myalgia $(36.7 \%)$, arthralgia $(35.0 \%)$, back pain $(32.3 \%)$, low back pain $(24.3 \%)$, and neck pain $(15.7 \%)$; the other COVID-19 symptoms were shortness of breath $(37.7 \%)$, loss of taste $(31.3 \%)$, loss of appetite $(30.0 \%)$, loss of smell $(27.0 \%)$, cough $(26.0 \%)$, headache $(16.7 \%)$, sore throat $(6.3 \%)$, fever $(4.0 \%)$, diarrhea $(4.0 \%)$, and dizziness $(2.0 \%)$, in the descending order of frequency (Fig. 1, Table 2).

At 1-month, $72.0 \%$ reported one or more symptoms, with $56.3 \%$ reported any musculoskeletal symptom and $51.0 \%$ reported any other COVID-19 symptom. The musculoskeletal symptoms were fatigue (44.3\% of patients reported), back pain $(22.7 \%)$, arthralgia $(22.0 \%)$, myalgia $(21.0 \%)$, low back pain $(16.3 \%)$, and neck pain $(10.3 \%)$; the other COVID19 symptoms were shortness of breath $(26.3 \%)$, loss of taste (15.0\%), cough $(14.0 \%)$, loss of smell (12.3\%), loss of appetite $(10.3 \%)$, headache $(8.7 \%)$, sore throat $(3.0 \%)$, diarrhea $(1.3 \%)$, dizziness $(1.3 \%)$, and fever $(0.3 \%)$, in the descending order of frequency (Fig. 1, Table 2).

The severity of musculoskeletal and other COVID19 symptoms at hospitalization, 2-week, and 1-month is detailed in Table 4.

After adjusting for other variables, neither age, nor sex, nor the length of hospital stay was associated with higher odds of persistence of fatigue, myalgia, and arthralgia at 1 month. After adjusting for age, sex, and length of hospital stay, increasing BMI was associated with higher odds of the persistence of fatigue (odds ratio $\{\mathrm{OR}\}$ with $95 \%$ confidence interval: $1.08,1.03$ to $1.13, p=0.003$ ), myalgia (OR: 1.08 , 1.01 to $1.14, p=0.015$ ), and arthralgia (OR: $1.07,1.02$ to $1.14, p=0.012$ ) at 1 month (Table 5).

\section{Discussion}

The study found that nearly three-quarters of patients reported one or more symptoms, with more than half of patients reported any musculoskeletal symptom at 1 month. The most common musculoskeletal symptom was fatigue (nearly half of patients), followed by back pain ( $\approx$ two in five), arthralgia ( $\approx$ two in five), myalgia ( $\approx$ two in five), low back pain, and neck pain; the most common other COVID19 symptom was shortness of breath ( $\approx$ a quarter of patients), followed by loss of taste, cough, loss of smell, loss of appetite, headache, sore throat, diarrhea, dizziness, and fever at 1 month. In addition, increasing BMI was associated with higher odds of the persistence of fatigue, myalgia, and arthralgia at 1-month; however, no association was found between age/sex/length of hospital stay and persistence of these symptoms.

The available information on the persistence of rheumatic and musculoskeletal symptoms after discharge with COVID-19 is scarce (Table 6) [12-15]. Carfi et al. analyzed 143 patients with a mean of 36 days after discharge. 
Table 1 Demographic, clinical, and COVID-19 characteristics of study population

\begin{tabular}{|c|c|}
\hline \multicolumn{2}{|l|}{ Characteristic } \\
\hline Age, years & $52.58 \pm 12.01$ \\
\hline Female sex & $121(40.3)$ \\
\hline BMI, $\mathrm{kg} / \mathrm{m}^{2}$ & $28.92 \pm 7.74$ \\
\hline \multicolumn{2}{|l|}{ Educational level } \\
\hline Illiterate & $8(2.7)$ \\
\hline Primary school & $104(34.7)$ \\
\hline Junior high school & $31(10.3)$ \\
\hline High school & $53(17.7)$ \\
\hline University & $104(34.7)$ \\
\hline Employed & $129(43.0)$ \\
\hline \multicolumn{2}{|l|}{ Smoking status } \\
\hline Current smoker & $22(7.3)$ \\
\hline Nonsmoker & $226(75.3)$ \\
\hline Ex-smoker & $52(17.3)$ \\
\hline Smoking, pack-years & $26.26 \pm 15.87$ \\
\hline \multicolumn{2}{|l|}{ Alcohol consumption status } \\
\hline Current use & $15(5.0)$ \\
\hline None & $285(95.0)$ \\
\hline \multicolumn{2}{|l|}{ Comorbidities } \\
\hline Any comorbidity & $195(65.0)$ \\
\hline Hypertension & $97(32.3)$ \\
\hline Diabetes mellitus & $85(28.3)$ \\
\hline Hyperlipidemia & $27(9.0)$ \\
\hline Coronary artery disease & $44(14.7)$ \\
\hline Thyroid disorders & $12(4.0)$ \\
\hline Renal disorders & $5(1.7)$ \\
\hline Asthma & $20(6.7)$ \\
\hline COPD & $7(2.3)$ \\
\hline Osteoarthritis & $8(2.7)$ \\
\hline Rheumatoid arthritis & $5(1.7)$ \\
\hline FMF & $4(1.3)$ \\
\hline SARS-CoV-2 RT-PCR test positivity & $262(87.3)$ \\
\hline COVID-19 chest CT findings & $270(90.0)$ \\
\hline Days from PCR test to hospitalization & $6.43 \pm 3.56$ \\
\hline Length of hospital stay, days & $7.60 \pm 3.95$ \\
\hline
\end{tabular}

The data are frequency (percentage) or mean \pm standard deviation $B M I$ body mass index, $C O P D$ chronic obstructive pulmonary disease, COVID-19 coronavirus disease 2019, CT computed tomography, $F M F$ familial Mediterranean Fever, $R T-P C R$ reverse-transcriptasepolymerase chain reaction, $S A R S-C o V-2$ Severe acute respiratory syndrome coronavirus 2

They found that $87.4 \%$ reported persistence of at least 1 symptom, particularly fatigue $(53.1 \%)$, shortness of breath (43.4\%), and joint pain, (27.3\%) [12]. Garrigues et al. surveyed 120 patients ( 96 ward patients and 24 intensive care patients) by phone, and found the most frequently reported persistent symptoms being fatigue (54.2\%), and shortness of breath (39.6\%) among ward patients [13]. In

Table 2 Frequency of hospitalization and postdischarge symptoms

\begin{tabular}{lccc}
\hline Characteristic & Hospitalization & 2 weeks & 1 month \\
\hline Any symptom & $300(100.0)$ & $260(86.7)$ & $216(72.0)$ \\
Any musculoskeletal & $277(92.3)$ & $218(72.7)$ & $169(56.3)$ \\
$\quad$ symptom & & & \\
Any other symptom & $296(98.7)$ & $217(72.3)$ & $153(51.0)$ \\
Fatigue & $262(87.3)$ & $192(64.0)$ & $133(44.3)$ \\
Myalgia & $190(63.3)$ & $110(36.7)$ & $63(21.0)$ \\
Arthralgia & $178(59.3)$ & $105(35.0)$ & $66(22.0)$ \\
Low back pain & $134(44.7)$ & $73(24.3)$ & $49(16.3)$ \\
Back pain & $162(54.0)$ & $97(32.3)$ & $68(22.7)$ \\
Neck pain & $80(26.7)$ & $47(15.7)$ & $31(10.3)$ \\
Fever & $196(65.3)$ & $12(4.0)$ & $1(0.3)$ \\
Cough & $203(67.7)$ & $78(26.0)$ & $42(14.0)$ \\
Loss of appetite & $215(71.7)$ & $90(30.0)$ & $31(10.3)$ \\
Shortness of breath & $230(76.7)$ & $113(37.7)$ & $79(26.3)$ \\
Diarrhea & $64(21.3)$ & $12(4.0)$ & $4(1.3)$ \\
Sore throat & $91(30.3)$ & $19(6.3)$ & $9(3.0)$ \\
Headache & $118(39.3)$ & $50(16.7)$ & $26(8.7)$ \\
Dizziness & $11(3.7)$ & $6(2.0)$ & $4(1.3)$ \\
Loss of taste & $159(53.0)$ & $94(31.3)$ & $45(15.0)$ \\
Loss of smell & $130(43.3)$ & $81(27.0)$ & $37(12.3)$ \\
\hline
\end{tabular}

The data are frequency (percentage). Percentages are among all patients $(n=300)$

Table 3 Location of myalgia and joint pain symptoms at hospitalization

\begin{tabular}{lc}
\hline Characteristic & \\
\hline Myalgia & \\
Present & $190(63.3)$ \\
Widespread & $120(40.0)$ \\
Local & $70(23.3)$ \\
Shoulder girdle & $7(10.0)$ \\
Arm & $11(15.7)$ \\
Thigh & $4(5.7)$ \\
Lower leg & $48(68.6)$ \\
Arthralgia & \\
Present & $178(59.3)$ \\
Widespread & $100(33.3)$ \\
Local & $78(26.0)$ \\
Jaw & $0(0.0)$ \\
Shoulder & $9(11.5)$ \\
Elbow & $2(2.6)$ \\
Wrist hand & $10(12.8)$ \\
Hip & $8(10.3)$ \\
Knee & $40(51.3)$ \\
Ankle foot & $9(11.5)$ \\
\hline
\end{tabular}

The data are frequency (percentage). Percentages are among all patients $(\mathrm{n}=300)$ except for regions, which are among the local myalgia/ arthralgia 
Table 4 Severity of hospitalization and postdischarge symptoms

\begin{tabular}{|c|c|c|c|c|c|c|c|}
\hline Characteristic & Hospitalization & 2 weeks & 1 month & Characteristic & Hospitalization & 2 weeks & 1 month \\
\hline Fatigue & & & & Mild & $25(8.3)$ & $29(9.7)$ & $22(7.3)$ \\
\hline None & $38(12.7)$ & $108(36.0)$ & $167(55.7)$ & Moderate & $29(9.7)$ & $28(9.3)$ & $6(2.0)$ \\
\hline Mild & $40(13.3)$ & $68(22.7)$ & $93(31.0)$ & Severe & $44(14.7)$ & $16(5.3)$ & $2(0.7)$ \\
\hline Moderate & $62(20.7)$ & $56(18.7)$ & $30(10.0)$ & Very severe & $117(39.0)$ & $17(5.7)$ & $1(0.3)$ \\
\hline Severe & $47(15.7)$ & $32(10.7)$ & $9(3.0)$ & Shortness of breath & & & \\
\hline Very severe & $113(37.7)$ & $36(12.0)$ & $1(0.3)$ & None & $70(23.3)$ & $187(62.3)$ & $221(73.7)$ \\
\hline Myalgia & & & & Mild & $50(16.7)$ & $52(17.3)$ & $67(22.3)$ \\
\hline None & $110(36.7)$ & $190(63.3)$ & $237(79.0)$ & Moderate & $47(15.7)$ & $34(11.3)$ & $10(3.3)$ \\
\hline Mild & $21(7.0)$ & $46(15.3)$ & $45(15.0)$ & Severe & $50(16.7)$ & $11(3.7)$ & $2(0.7)$ \\
\hline Moderate & $60(20.0)$ & $36(12.0)$ & $12(4.0)$ & Very severe & $83(27.7)$ & $16(5.3)$ & - \\
\hline Severe & $33(11.0)$ & $6(2.0)$ & $3(1.0)$ & Diarrhea & & & \\
\hline Very severe & $76(25.3)$ & $22(7.3)$ & $3(1.0)$ & None & $236(78.7)$ & $288(96.0)$ & $296(98.7)$ \\
\hline Arthralgia & & & & Mild & $30(10.0)$ & $3(1.0)$ & $3(1.0)$ \\
\hline None & $122(40.7)$ & $195(65.0)$ & $234(78.0)$ & Moderate & $15(5.0)$ & $6(2.0)$ & - \\
\hline Mild & $26(8.7)$ & $36(12.0)$ & $46(15.3)$ & Severe & $13(4.3)$ & $1(0.3)$ & - \\
\hline Moderate & $54(18.0)$ & $38(12.7)$ & $15(5.0)$ & Very severe & $6(2.0)$ & $2(0.7)$ & $1(0.3)$ \\
\hline Severe & $29(9.7)$ & $10(3.3)$ & $3(1.0)$ & Sore throat & & & \\
\hline Very severe & $69(23.0)$ & $21(7.0)$ & $2(0.7)$ & None & 209 (69.7) & $281(93.7)$ & $291(97.0)$ \\
\hline Low back pain & & & & Mild & $58(19.3)$ & $10(3.3)$ & $6(2.0)$ \\
\hline None & $166(55.3)$ & $227(75.7)$ & $251(83.7)$ & Moderate & $19(6.3)$ & $5(1.7)$ & $3(1.0)$ \\
\hline Mild & $19(6.3)$ & $31(10.3)$ & 35 (11.7) & Severe & $8(2.7)$ & $3(1.0)$ & - \\
\hline Moderate & $30(10.0)$ & $19(6.3)$ & $10(3.3)$ & Very severe & $6(2.0)$ & $1(0.3)$ & - \\
\hline Severe & $25(8.3)$ & $7(2.3)$ & $2(0.7)$ & Headache & & & \\
\hline Very severe & $60(20.0)$ & $16(5.3)$ & $2(0.7)$ & None & $182(60.7)$ & $250(83.3)$ & $274(91.3)$ \\
\hline Back pain & & & & Mild & $19(6.3)$ & $18(6.0)$ & $14(4.7)$ \\
\hline None & $138(46.0)$ & $203(67.7)$ & $232(77.3)$ & Moderate & $28(9.3)$ & $19(6.3)$ & $12(4.0)$ \\
\hline Mild & $32(10.7)$ & $43(14.3)$ & $55(18.3)$ & Severe & $24(8.0)$ & $11(3.7)$ & - \\
\hline Moderate & $42(14.0)$ & $24(8.0)$ & $9(3.0)$ & Very severe & $47(15.7)$ & $2(0.7)$ & - \\
\hline Severe & $31(10.3)$ & $10(3.3)$ & $2(0.7)$ & Dizziness & & & \\
\hline Very severe & $57(19.0)$ & $20(6.7)$ & $2(0.7)$ & None & $289(96.3)$ & 294 (98.0) & $296(98.7)$ \\
\hline Neck pain & & & & Mild & $1(0.3)$ & $3(1.0)$ & $2(0.7)$ \\
\hline None & $220(73.3)$ & $253(84.3)$ & 269 (89.7) & Moderate & $5(1.7)$ & $1(0.3)$ & $2(0.7)$ \\
\hline Mild & $13(4.3)$ & $23(7.7)$ & $23(7.7)$ & Severe & $4(1.3)$ & $2(0.7)$ & - \\
\hline Moderate & $23(7.7)$ & $12(4.0)$ & $5(1.7)$ & Very severe & $1(0.3)$ & - & - \\
\hline Severe & $19(6.3)$ & $4(1.3)$ & $1(0.3)$ & Loss of taste & & & \\
\hline Very severe & $25(8.3)$ & $8(2.7)$ & $2(0.7)$ & None & $141(47.0)$ & $206(68.7)$ & $255(85.0)$ \\
\hline Fever & & & & Mild & $20(6.7)$ & $19(6.3)$ & $26(8.7)$ \\
\hline None & $104(34.7)$ & $288(96.0)$ & 299 (99.7) & Moderate & $14(4.7)$ & $21(7.0)$ & $10(3.3)$ \\
\hline Mild & $53(17.7)$ & $4(1.3)$ & $1(0.3)$ & Severe & $22(7.3)$ & $27(9.0)$ & $8(2.7)$ \\
\hline Moderate & $63(21.0)$ & $6(2.0)$ & - & Very severe & $103(34.3)$ & $27(9.0)$ & $1(0.3)$ \\
\hline Severe & $56(18.7)$ & $1(0.3)$ & - & Loss of smell & & & \\
\hline Very severe & $24(8.0)$ & $1(0.3)$ & - & None & $170(56.7)$ & $219(73.0)$ & $263(87.7)$ \\
\hline Cough & & & & Mild & $15(5.0)$ & $22(7.3)$ & $22(7.3)$ \\
\hline None & $97(32.3)$ & $222(74.0)$ & $258(86.0)$ & Moderate & $13(4.3)$ & $17(5.7)$ & $7(2.3)$ \\
\hline Mild & $72(24.0)$ & 35 (11.7) & $32(10.7)$ & Severe & $16(5.3)$ & $17(5.7)$ & $7(2.3)$ \\
\hline Moderate & $37(12.3)$ & $24(8.0)$ & $9(3.0)$ & Very severe & $86(28.7)$ & $25(8.3)$ & $1(0.3)$ \\
\hline Severe & $34(11.3)$ & $13(4.3)$ & $1(0.3)$ & Characteristic & & & \\
\hline Very severe & $60(20.0)$ & $6(2.0)$ & - & Myalgia & & & \\
\hline Loss of appetite & & & & Present & & $190(63.3)$ & \\
\hline None & $85(28.3)$ & $210(70.0)$ & 269 (89.7) & Widespread & & $120(40.0)$ & \\
\hline
\end{tabular}

Table 4 (continued) 
Table 4 (continued)

\begin{tabular}{|c|c|c|c|}
\hline Characteristic & Hospitalization & 2 weeks & 1 month \\
\hline Local & & $70(23.3)$ & \\
\hline Shoulder girdle & & $7(10.0)$ & \\
\hline Arm & & $11(15.7)$ & \\
\hline Thigh & & $4(5.7)$ & \\
\hline Lower leg & & 48 (68.6) & \\
\hline \multicolumn{4}{|l|}{ Arthralgia } \\
\hline Present & & $178(59.3)$ & \\
\hline Widespread & & $100(33.3)$ & \\
\hline Local & & $78(26.0)$ & \\
\hline Jaw & & $0(0.0)$ & \\
\hline Shoulder & & $9(11.5)$ & \\
\hline Elbow & & $2(2.6)$ & \\
\hline Wrist hand & & $10(12.8)$ & \\
\hline Hip & & $8(10.3)$ & \\
\hline Knee & & $40(51.3)$ & \\
\hline Ankle foot & & $9(11.5)$ & \\
\hline
\end{tabular}

The data are frequency (percentage). Percentages are among all patients $(n=300)$

another phone survey, Halpin et al. analyzed postdischarge symptoms in 100 participants (68 ward patients and 32 intensive care patients). Fatigue was the most common reported symptom by $60.3 \%$ followed by shortness of breath by $42.6 \%$ in the ward group [14]. Arnold et al. analyzed 110 patients (both ward and intensive care patients) and found that $73.6 \%$ of patients reported at least one ongoing symptom with $39.1 \%$ shortness of breath, $39.1 \%$ fatigue, and $22.7 \%$ myalgia [15]. Interestingly, in each study, the most common two persistent symptoms were fatigue $(39 \%-60 \%)$ and shortness of breath $(39 \%-43 \%)$ [12-15]; the present results (the most common fatigue by
$44 \%$ followed by shortness of breath by $26 \%$ ) are consistent with those of the previous studies. In addition, the present study provides information on the persistence of other musculoskeletal symptoms: back pain, arthralgia, and myalgia each was present in two-fifths of patients, and a less frequently low back pain and neck pain were persisted. Regarding the other COVID-19 symptoms, loss of taste, cough, loss of smell, and loss of appetite each was persisted in at least one-tenth of patients. The wide spectrum of persistent symptoms requires multidisciplinary care for discharged survivors. Considering the relatively high frequency of rheumatic and musculoskeletal symptoms, rheumatologists should be involved in multidisciplinary teams caring for COVID-19 survivors.

A meta-analysis addressing the association of obesity and COVID-19 showed individuals with obesity were more at risk for COVID-19 positive, hospitalization, intensive care admission, and mortality [20]. The severity of COVID-19 in obese patients has been attributed to metabolic dysfunction, immune response impairments, adipose inflammation, and decreased lung function [20, 21]. Furthermore, in a phone survey study investigating the persistent symptoms 14-21 days after testing among the 274 symptomatic outpatients (not hospitalized), obesity was associated with not returning to the usual health [22]. In the present study, increasing BMI was associated with higher odds of the persistence of fatigue, myalgia, and arthralgia in hospitalized COVID-19 patients.

Several mechanisms have been proposed for rheumatic and musculoskeletal symptoms in COVID-19 [23-27]. The acute rheumatic and musculoskeletal symptoms of COVID-19 (i.e. myalgia, fatigue, arthralgia) have been widely attributed to immune response and pro-inflammatory cytokines generated after infection [23-27]. Beyond systemic immune response/inflammation, a direct invasion/

Table 5 Association of age, sex, BMI, and length of hospital stay with presence of fatigue, myalgia, and arthralgia at 1-month

\begin{tabular}{|c|c|c|c|c|c|c|}
\hline \multirow[t]{2}{*}{ Characteristic } & \multicolumn{2}{|l|}{ Fatigue } & \multicolumn{2}{|l|}{ Myalgia } & \multicolumn{2}{|l|}{ Arthralgia } \\
\hline & Unadjusted OR & Adjusted OR & Unadjusted OR & Adjusted OR & Unadjusted OR & Adjusted OR \\
\hline Age & $\begin{array}{l}0.98(0.96-1.00) \\
p=0.081\end{array}$ & $\begin{array}{l}0.98(0.96-1.00) \\
p=0.060\end{array}$ & $\begin{array}{l}1.01(0.99-1.03) \\
p=0.551\end{array}$ & $\begin{array}{l}1.01(0.98-1.03) \\
p=0.613\end{array}$ & $\begin{array}{l}1.00(0.98-1.02) \\
p=0.833\end{array}$ & $\begin{array}{l}1.00(0.98-1.02) \\
p=0.957\end{array}$ \\
\hline Female sex & $\begin{array}{l}1.51(0.95-2.41) \\
p=0.082\end{array}$ & $\begin{array}{l}1.42(0.89-2.28) \\
p=0.145\end{array}$ & $\begin{array}{l}2.02(1.15-3.54) \\
p=0.014\end{array}$ & $\begin{array}{l}1.79(1.00-3.20) \\
p=0.052\end{array}$ & $\begin{array}{l}1.42(0.82-2.46) \\
p=0.214\end{array}$ & $\begin{array}{l}1.26(0.71-2.24) \\
p=0.429\end{array}$ \\
\hline BMI & $\begin{array}{l}\text { 1.08 (1.03-1.13) } \\
p=0.003\end{array}$ & $\begin{array}{l}1.08(1.03-1.13) \\
p=0.003\end{array}$ & $\begin{array}{l}1.09(1.03-1.16) \\
p=0.005\end{array}$ & $\begin{array}{l}1.08(1.01-1.14) \\
p=0.015\end{array}$ & $\begin{array}{l}1.08(1.02-1.15) \\
p=0.007\end{array}$ & $\begin{array}{l}1.07(1.02-1.14) \\
p=0.012\end{array}$ \\
\hline Length of stay & $\begin{array}{l}0.96(0.90-1.02) \\
p=0.158\end{array}$ & $\begin{array}{l}0.98(0.92-1.04) \\
p=0.468\end{array}$ & $\begin{array}{l}0.96(0.88-1.03) \\
p=0.236\end{array}$ & $\begin{array}{l}0.96(0.88-1.04) \\
p=0.283\end{array}$ & $\begin{array}{l}0.98(0.91-1.05) \\
p=0.529\end{array}$ & $\begin{array}{l}0.98(0.91-1.06) \\
p=0.628\end{array}$ \\
\hline
\end{tabular}

Bold values denote statistical significance $(p$ values $<0.05)$

Generalized estimating equations with binary logistic model was used. In adjusted models for age, sex, BMI, and length of hospital stay, the other three were adjusted

$B M I$ Body mass index, OR Odds ratio 
Table 6 Frequency of postdischarge symptoms in the literature

\begin{tabular}{|c|c|c|c|c|c|}
\hline & Carfi et al. $[12]^{\dagger}$ & Garrigues et al. [13] & Halpin et al. $[14]^{\dagger}$ & Arnold et al. $[15]^{\ddagger}$ & Present study* \\
\hline Country & Italy & France & UK & UK & Turkey \\
\hline Number of total patients & 143 & 120 & 100 & 110 & 300 \\
\hline Number of ward patients & $125(87.4 \%)$ & $96(80.0 \%)$ & $68(68.0 \%)$ & N/A & $300(100.0 \%)$ \\
\hline Number of intensive care patients & $18(12.6 \%)$ & $24(20.0 \%)$ & $32(32.0 \%)$ & N/A & 0 \\
\hline Days after hospital admission & N/A & 111 & N/A & 83 & 30 \\
\hline Days after discharge & 36 & N/A & 48 & N/A & N/A \\
\hline Age, years & $56.5 \pm 14.6$ & $64.1 \pm 16.1$ & $70.5(20-93)$ & $60(44-76)$ & $52.8 \pm 12.0$ \\
\hline Female sex & $53(37.1 \%)$ & $40(41.7 \%)$ & $33(48.5 \%)$ & $42(38.2 \%)$ & $121(40.3 \%)$ \\
\hline Any symptom & $87.4 \%$ & N/A & N/A & $73.6 \%$ & $72.0 \%$ \\
\hline Any musculoskeletal symptom & N/A & N/A & N/A & N/A & $56.3 \%$ \\
\hline Fatigue & $53.1 \%$ & $54.2 \%$ & $60.3 \%$ & $39.1 \%$ & $44.3 \%$ \\
\hline Myalgia & $\mathrm{N} / \mathrm{A}^{\S}$ & N/A & N/A & $22.7 \%$ & $21.0 \%$ \\
\hline Arthralgia & $27.3 \%$ & N/A & N/A & $4.5 \%$ & $22.0 \%$ \\
\hline Low back pain & N/A & N/A & N/A & N/A & $16.3 \%$ \\
\hline Back pain & N/A & N/A & N/A & N/A & $22.7 \%$ \\
\hline Neck pain & N/A & N/A & N/A & N/A & $10.3 \%$ \\
\hline Any other symptom & N/A & N/A & N/A & N/A & $51.0 \%$ \\
\hline Fever & N/A & N/A & N/A & $0.9 \%$ & $0.3 \%$ \\
\hline Cough & $N / A^{\S}$ & $14.6 \%$ & $\mathrm{~N} / \mathrm{A}^{\S}$ & $11.8 \%$ & $14.0 \%$ \\
\hline Loss of appetite & $\mathrm{N} / \mathrm{A}^{\S}$ & N/A & N/A & N/A & $10.3 \%$ \\
\hline Shortness of breath & $43.4 \%$ & $39.6 \%$ & $42.6 \%$ & $39.1 \%$ & $26.3 \%$ \\
\hline Diarrhea & $\mathrm{N} / \mathrm{A}^{\S}$ & N/A & N/A & $0.9 \%$ & $1.3 \%$ \\
\hline Sore throat & $\mathrm{N} / \mathrm{A}^{\S}$ & N/A & N/A & N/A & $3.0 \%$ \\
\hline Headache & $\mathrm{N} / \mathrm{A}^{\S}$ & N/A & N/A & $1.8 \%$ & $8.7 \%$ \\
\hline Dizziness & N/A & N/A & N/A & N/A & $1.3 \%$ \\
\hline Loss of taste & $N / A^{\S}$ & $9.4 \%$ & N/A & N/A & $15.0 \%$ \\
\hline Loss of smell & N/A & $14.6 \%$ & N/A & $11.8 \%$ & $12.3 \%$ \\
\hline
\end{tabular}

The data are frequency, frequency (percentage), mean, median, mean \pm standard deviation, median (range) or median (interquartiles)

N/A not available

*1-month data are presented for symptoms

${ }^{\dagger}$ The data were presented among total patients (both ward and intensive care)

The data were presented among ward patients

${ }^{\S}$ The data were presented as a bar chart; therefore, exact value could not be obtained

injury of musculoskeletal cells by SARS-CoV-2 through the angiotensin-converting enzyme 2 (ACE2) receptor is the other proposed hypothesis [23-27]. Future studies aimed at elucidating the mechanisms underlying the persistent symptoms are needed.

\section{Limitations}

The present study has some limitations. The main limitation was that it had no control group of patients hospitalized for conditions other than COVID-19. A design with a control group would have managed to allow a better interpretation of the present results. Moreover, it included patients who were hospitalized for COVID-19; therefore, the results cannot be generalized to nonhospitalized patients. Also, as COVID-19 patients who have more comorbid conditions have higher odds of hospitalization [10, 28, 29], these comorbidities might have influenced the results observed in the present study as confounding factors. Furthermore, the study design does not allow interpreting the causality between BMI and persistence of fatigue, myalgia, and arthralgia. Despite these limitations, this study provides detailed data on persistence and risk factors of rheumatic and musculoskeletal symptoms after discharge with COVID-19. This information may lead to better address/care for the persistent rheumatic and musculoskeletal symptoms in COVID-19 survivors. 


\section{Conclusion}

Nearly three-quarters of patients reported one or more symptoms, with more than half of patients, who were hospitalized for COVID-19, reported any musculoskeletal symptom at 1 month. The most common musculoskeletal symptom was fatigue, followed by back pain, arthralgia, myalgia, low back pain, and neck pain at 1-month. The persistence of fatigue, myalgia, and arthralgia was related to BMI. The study results increase our understanding of the spectrum of COVID-19, which, in turn, may lead to more efficient and better care for COVID-19 survivors.

Supplementary Information The online version contains supplementary material available at https://doi.org/10.1007/s00296-021-04882-8.

Author contributions Design of the study: FK, FDG; data collection: FK; data analysis: SK; data interpretation: FK, FDG, SK; writing the initial draft: SK; critical revision of the article: FK, FDG, SK; final approval of the version of the article to be published: FK, FDG, SK.

Funding None.

\section{Declarations}

Conflict of interest The authors declare no conflicts of interest.

Ethical approval The ethical approval for the study was granted by the Ethics Committee of the Gulhane Scientific Researches, University of Health Sciences (No: 2020-403).

\section{References}

1. Kardeş S, Kuzu AS, Raiker R, Pakhchanian H, Karagülle M (2021) Public interest in rheumatic diseases and rheumatologist in the United States during the COVID-19 pandemic: evidence from Google Trends. Rheumatol Int 41:329-334. https://doi.org/ 10.1007/s00296-020-04728-9

2. Coskun Benlidayi I, Kurtaran B, Tirasci E, Guzel R (2020) Coronavirus disease 2019 (COVID-19) in a patient with ankylosing spondylitis treated with secukinumab: a case-based review. Rheumatol Int 40:1707-1716. https://doi.org/10.1007/ s00296-020-04635-z

3. Durcan G, Barut K, Haslak F, Doktur H, Yildiz M, Adrovic A, Sahin S, Kasapcopur O (2021) Psychosocial and clinical effects of the COVID-19 pandemic in patients with childhood rheumatic diseases and their parents. Rheumatol Int 41:575-583. https://doi. org/10.1007/s00296-021-04790-x

4. Kardeş S, Erdem A, Gürdal H. Public Interest in Musculoskeletal Symptoms and Disorders during the COVID-19 Pandemic: Infodemiology Study. 2021. Z Rheumatol. https://doi.org/10.1007/ s00393-021-00989-2.

5. Cleaton N, Raizada S, Barkham N, Venkatachalam S, Sheeran TP, Adizie T, Sapkota H, Singh BM, Bateman J (2021) The impact of COVID-19 on rheumatology patients in a large UK centre using an innovative data collection technique: prevalence and effect of social shielding. Rheumatol Int 41:707-714. https://doi.org/10. 1007/s00296-021-04797-4
6. Velikova T, Georgiev T (2021) SARS-CoV-2 vaccines and autoimmune diseases amidst the COVID-19 crisis. Rheumatol Int 41:509-518. https://doi.org/10.1007/s00296-021-04792-9

7. Andreica I, Guminski B, Sokolar J, Patru A, Baraliakos X, Braun J (2021) Drohende Corona-Endemie in einer rheumatologischen Spezialklinik - relative Entwarnung durch konsequente Testung [Threat of a SARS-CoV-2 endemic in a large hospital specialized in rheumatic diseases-relative all clear through consistent testing]. Z Rheumatol 80:45-47. https://doi.org/10.1007/ s00393-021-00959-8

8. Batu ED, Özen S (2020) Implications of COVID-19 in pediatric rheumatology. Rheumatol Int 40:1193-1213. https://doi.org/10. 1007/s00296-020-04612-6

9. WHO Coronavirus Disease (COVID-19) Dashboard. https://covid 19.who.int/. Accessed 4 March 2021

10. Stokes EK, Zambrano LD, Anderson KN, et al (2020) Coronavirus Disease 2019 Case Surveillance - United States, January 22-May 30, 2020. MMWR Morb Mortal Wkly Rep 69:759-765. https://doi.org/10.15585/mmwr.mm6924e2.

11. Ciaffi J, Meliconi R, Ruscitti P, Berardicurti O, Giacomelli R, Ursini F (2020) Rheumatic manifestations of COVID-19: a systematic review and meta-analysis. BMC Rheumatol 28(4):65. https://doi.org/10.1186/s41927-020-00165-0

12. Carfì A, Bernabei R, Landi F; Gemelli Against COVID-19 PostAcute Care Study Group (2020) Persistent Symptoms in Patients After Acute COVID-19. JAMA 324:603-605. https://doi.org/10. 1001/jama.2020.12603

13. Garrigues E, Janvier P, Kherabi Y, Le Bot A, Hamon A, Gouze H, Doucet L, Berkani S, Oliosi E, Mallart E, Corre F, Zarrouk V, Moyer JD, Galy A, Honsel V, Fantin B, Nguyen Y (2020) Postdischarge persistent symptoms and health-related quality of life after hospitalization for COVID-19. J Infect 81:e4-e6. https://doi. org/10.1016/j.jinf.2020.08.029

14. Halpin SJ, McIvor C, Whyatt G, Adams A, Harvey O, McLean L, Walshaw C, Kemp S, Corrado J, Singh R, Collins T, O'Connor RJ, Sivan M (2021) Postdischarge symptoms and rehabilitation needs in survivors of COVID-19 infection: A cross-sectional evaluation. J Med Virol 93:1013-1022. https://doi.org/10.1002/jmv.26368

15. Arnold DT, Hamilton FW, Milne A, Morley AJ, Viner J, Attwood M, Noel A, Gunning S, Hatrick J, Hamilton S, Elvers KT, Hyams C, Bibby A, Moran E, Adamali HI, Dodd JW, Maskell NA, Barratt SL (2020) Patient outcomes after hospitalisation with COVID19 and implications for follow-up: results from a prospective UK cohort. Thorax 76:399-401. https://doi.org/10.1136/thora xjnl-2020-216086

16. Moreno-Pérez O, Merino E, Leon-Ramirez JM, Andres M, Ramos JM, Arenas-Jiménez J, Asensio S, Sanchez R, Ruiz-Torregrosa P, Galan I, Scholz A, Amo A, González-delaAleja P, Boix V, Gil J; COVID19-ALC research group (2021) Post-acute COVID-19 syndrome. Incidence and risk factors: A Mediterranean cohort study. J Infect S0163-4453(21)00009-8. https://doi.org/10.1016/j. jinf.2021.01.004.

17. Carvalho-Schneider C, Laurent E, Lemaignen A, Beaufils E, Bourbao-Tournois C, Laribi S, Flament T, Ferreira-Maldent N, Bruyère F, Stefic K, Gaudy-Graffin C, Grammatico-Guillon L, Bernard L (2021) Follow-up of adults with noncritical COVID-19 two months after symptom onset. Clin Microbiol Infect 27:258 263. https://doi.org/10.1016/j.cmi.2020.09.052

18. Bujang MA, Sa'at N, Sidik TMITAB, Joo LC (2018) Sample Size Guidelines for Logistic Regression from Observational Studies with Large Population: Emphasis on the Accuracy Between Statistics and Parameters Based on Real Life Clinical Data. Malays J Med Sci 25:122-130. https://doi.org/10.21315/mjms2018.25.4. 12. 
19. Misra DP, Zimba O, Gasparyan AY (2021) Statistical data presentation: a primer for rheumatology researchers. Rheumatol Int 41:43-55. https://doi.org/10.1007/s00296-020-04740-z

20. Popkin BM, Du S, Green WD, Beck MA, Algaith T, Herbst CH, Alsukait RF, Alluhidan M, Alazemi N, Shekar M (2020) Individuals with obesity and COVID-19: A global perspective on the epidemiology and biological relationships. Obes Rev 21:e13128. https://doi.org/10.1111/obr.13128

21. Sattar N, McInnes IB, McMurray JJV (2020) Obesity Is a Risk Factor for Severe COVID-19 Infection: Multiple Potential Mechanisms. Circulation 142:4-6. https://doi.org/10.1161/CIRCU LATIONAHA.120.047659

22. Tenforde MW, Kim SS, Lindsell CJ, Billig Rose E, Shapiro NI, Files DC, Gibbs KW, Erickson HL, Steingrub JS, Smithline HA, Gong MN, Aboodi MS, Exline MC, Henning DJ, Wilson JG, Khan A, Qadir N, Brown SM, Peltan ID, Rice TW, Hager DN, Ginde AA, Stubblefield WB, Patel MM, Self WH, Feldstein LR; IVY Network Investigators; CDC COVID-19 Response Team; IVY Network Investigators (2020) Symptom Duration and Risk Factors for Delayed Return to Usual Health Among Outpatients with COVID-19 in a Multistate Health Care Systems Network United States, March-June 2020. MMWR Morb Mortal Wkly Rep 69:993-998. https://doi.org/10.15585/mmwr.mm6930e1

23. Disser NP, De Micheli AJ, Schonk MM, Konnaris MA, Piacentini AN, Edon DL, Toresdahl BG, Rodeo SA, Casey EK, Mendias CL (2020) Musculoskeletal Consequences of COVID-19. J Bone Joint Surg Am 15(102):1197-1204. https://doi.org/10.2106/JBJS.20. 00847

24. Ramani SL, Samet J, Franz CK, Hsieh C, Nguyen CV, Horbinski C, Deshmukh S (2021) Musculoskeletal involvement of
COVID-19: review of imaging. Skeletal Radiol. https://doi.org/ 10.1007/s00256-021-03734-7

25. Ahmed S, Zimba O, Gasparyan AY (2021) COVID-19 and the clinical course of rheumatic manifestations. Clin Rheumatol. https://doi.org/10.1007/s10067-021-05691-x

26. Ahmed S, Anirban P, Lawrence A (2020) Decoding the Pathophysiology of COVID-19: An Unified Hypothesis for All Major Manifestations. https://ssrn.com/abstract=3603361. https://doi. org/10.2139/ssrn.3603361. (Preprint)

27. Shah S, Danda D, Kavadichanda C, Das S, Adarsh MB, Negi VS (2020) Autoimmune and rheumatic musculoskeletal diseases as a consequence of SARS-CoV-2 infection and its treatment. Rheumatol Int 40:1539-1554. https://doi.org/10.1007/ s00296-020-04639-9

28. Ahmed S, Gasparyan AY, Zimba O (2021) Comorbidities in rheumatic diseases need special consideration during the COVID-19 pandemic. Rheumatol Int 41:243-256. https://doi.org/10.1007/ s00296-020-04764-5

29. Price-Haywood EG, Burton J, Fort D, Seoane L (2020) Hospitalization and Mortality among Black Patients and White Patients with Covid-19. N Engl J Med 25(382):2534-2543. https://doi.org/ 10.1056/NEJMsa2011686

Publisher's Note Springer Nature remains neutral with regard to jurisdictional claims in published maps and institutional affiliations. 